\title{
PERFIL DE CONSUMO DE ALIMENTOS DURANTE LA JORNADA ESCOLAR EN UNA COMUNIDAD EDUCATIVA VULNERABILIZADA DE LA CIUDAD DE CÓRDOBA, ARGENTINA.
}

\author{
FOOD CONSUMPTION PROFILE DURING \\ SCHOOL DAY IN A VULNERABLE SCHOOL \\ COMMUNITY IN THE CITY OF CORDOBA, \\ ARGENTINA.
}

PERFIL DO CONSUMO DE ALIMENTOS DURANTE UMA JORNADA ESCOLAR EM UMA COMUNIDADE EDUCACIONAL VULNERÁVEL DA CIDADE DE CÓRDOBA, ARGENTINA

Giordano, María S. ${ }^{1}$

Barnetche, María M. ${ }^{2}$

Álvarez, Pedro S. ${ }^{3}$

Cornejo, Lila S. ${ }^{4}$

'Licenciada en Psicología. Prof. Asistente de Psicología Evolutiva Facultad de Odontología. UNC.

2Odontóloga, Doctorando.

Mgter. En Salud Pública.

Facultad de Odontología. UNC.

${ }^{3}$ Licenciado en Psicología.

Prof. Asistente de Psicología

Evolutiva Facultad de

Odontología. UNC.

${ }^{4}$ Dra. en Ciencias Biológicas.

Prof. Tít. Biología Celular.

Facultad de Odontología. UNC.

Trabajo recibido: 20 de Noviembre 2016 Aprobado: 14 de Marzo 2016.

\section{Resumen}

La alimentación es una de las dimensiones fuertemente involucrada en los procesos de salud enfermedad atención. Con el objetivo de conocer el perfil de consumo de alimentos durante la jornada escolar en una comunidad educativa vulnerabilizada, se desarrolló un estudio transversal cuanti-cualitativo. Para la recolección de datos se realizó, durante una semana escolar en ambos turnos: registros escritos de observación participante de recreos y comedor y del menú del comedor y registro fotográfico de los productos ofertados en kioscos (interno y externo a la escuela) y de residuos contenidos en basureros. Los resultados obtenidos destacan un marcado predominio de alimentos del grupo de dulces y azúcares en la oferta y en las preferencias de consumo de los niños durante la jornada escolar. Existen alimentos que se repiten en el consumo por parte de los docentes, lo cual de alguna manera estaría presentándose como un refuerzo sistemático al hábito de consumo de los niños.

Palabras clave: preferencias alimentarias, hábitos alimenticios; consumo de alimentos 
Eating is one of the dimensions which isstrongly involved in thehealth-sickness-care process. In order to know the food consumption profile during school day in a vulnerable school community, a quanty-qualitative cross-sectional study was developed. Data were collected during one school week, in both shifts: through written records of participant observation of breaks and canteen and canteen menu; and photographic record of products offered in kiosks (inside and outside the school) and trash found in trashcans. Results show high prevalence of food from the sweet and sugar group in the offer as well as in children's consumption choice during school hours. There are some food items which are also eaten by teachers; which somehow is a systematic reinforcement to the kids consumption habits.

Key words: eating preferences, eating habits, food consumption.

\section{Resumo}

A alimentação é uma das dimensões fortemente envolvida nos processos de cuidados de saúde-doença. A fim de conhecer o perfil de consumo de alimentos durante uma jornada escolar em uma comunidade educativa vulnerável, desenvolveu-se, um estudo transversal, quanti-qualitativo. Para a coleta de dados foi realizada, durante uma semana escolar em dois turnos: registos escritos de observação participante dos intervalos de recreio, do refeitório e do cardápio do refeitório, e registro fotográfico dos produtos oferecidos nos quiosques (internos e externos à escola) e resíduos contido em lixeiros. Os resultados salientam um predomínio destacável de alimentos do grupo dos doces e açúcares na oferta e de preferências de consumo das crianças durante a jornada escolar. Existem alimentos que são repetidos no consumo por parte dos professores, o que de alguma forma poderia considerar-se como um reforço sistemático dos hábitos de consumo das crianças.

Palavras-chave: preferências alimentares, hábitos alimentares; consumo de alimentos.

\section{Introducción}

Una de las inequidades vividas en las zonas urbano-marginales de las grandes ciudades del MERCOSUR, es la que se expresa en términos de salud de la población. En estos sectores las escuelas y los centros comunitarios son instituciones de importante presencia que pueden constituirse en espacios de promoción de salud.

El desarrollo de estrategias contextualizadas, que posibiliten la comprensión integral del proceso salud-enfermedad-atención, exige una propuesta teórica y metodológica que excede a las capacidades propias de las distintas disciplinas del área de la salud y requiere una elaboración cooperativa e interdisciplinaria que permita el abordaje desde la complejidad de dicho proceso.

Desde lo que denominamos promoción contextualizada, cobran interés las interrelaciones entre las dimensiones biológicas y socioculturales de estas comunidades, que se expresan en las representaciones y prácticas de salud, la frecuencia y distribución de las problemáticas de salud, las pautas de consumo de alimentos, la accesibilidad a la atención médico-odontológica, las condiciones ambientales, en la infraestructura, en el acceso a la educación, etc. todas ellas en relación con el estado de salud de la comunidad.

En el marco del proyecto "Promoción contextualizada de la salud en comunidades vulnerables" (1) resulta de interés conocer, cómo se manifiestan e interrelacionan dichas dimensiones en comunidades en situación de vulnerabilidad, a fin de desarrollar e implementar estrategias de promoción de salud, que respondan al circuito de necesidades y potencialidades de la comunidad.

Por su parte la jornada escolar ha sido objeto de varios estudios $(2,3)$ en lo que respecta 
a las pautas alimentarias o al consumo de alimentos por parte de niños o de los actores de la institución escolar. En este escenario, los hábitos alimentarios son el resultado de la interrelación entre las prácticas y el significado atribuido a las mismas según Moncunill, Bella y Cornejo (4). Pudiéndose plantearse una relación entre consumo de alimentos y salud bucal en general.

El consumo de alimentos de los niños en la escuela preferentemente es de grasas, azúcares y sodio, con escaso valor nutricional para el alumno (5). El principal grupo de alimentos en esta condición está representado por golosinas, gaseosas y snacks (6). Este consumo se ve principalmente en el tiempo de recreación en la jornada escolar, en concordancia con lo informado en un estudio sobre colaciones de niños de escuelas municipalizadas en el que se refiere que en el contexto regional las pautas alimentarias de los niños escolarizados son deficitarias, principalmente de bajo consumo de lácteos, verduras, frutas y pescado (7). Un hecho que se destaca es el manejo del dinero que tienen los niños para consumir en los recreos y la similitud de los productos de colación que traen de sus casas con los que ofrecen los kioscos o tiendas escolares. Por lo general estos espacios ofrecen alimentos con alto contenido energético y calórico Así mismo los productos más publicitados no son necesariamente los más nutricios $(8,9)$. Tal perspectiva podría contribuir a una pauta de alimentación deficitaria para el desarrollo óptimo del niño como así también a la creciente obesidad que podría observarse.

Al respecto, se ha estudiado que la pauta de ingesta o consumo de alimentos en diferentes contextos depende de: el entorno de la persona y de sus grupos significativos, la familia, instituciones educativas, condiciones económicas culturales, y las políticas públicas (2). Según lo anterior, se encontrarían afecciones sistemáticas en la boca relacionadas con deficiencias nutricionales y con lo cariogenico de algunos alimentos. Como también disfunciones en praxias masticatorias y deglución con molestias y dolor (2).

Es necesario diseñar estrategias contextualizadas, que contribuyan a la comprensión integral del proceso salud-enfermedad-atención, atendiendo la complejidad de dicho proceso desde una propuesta teórica y metodológica interdisciplinaria que supere las capacidades propias de las distintas disciplinas del área de la salud. Para no solo enfocar las variables epidemiológicas sino buscar la complementariedad con los procesos biológicos y socioculturales (2).

Por lo tanto, se estima que el consumo de alimentos esta relacionado de manera particular con las pautas culturales aprendidas por el sujeto en interacción con su contexto y aprendizajes particulares.

En ese sentido, el presente trabajo tuvo como objetivo conocer el perfil de consumo de alimentos durante la jornada escolar en la escuela "María Tránsito Cabanillas" de barrio El Quebracho de la ciudad de Córdoba, Argentina.

\section{Materiales y Métodos}

Se desarrolló un estudio transversal cuanti-cualitativo, en ambos turnos desde el 8 al 12 de Abril de 2013, en la escuela "MARIA TRANSITO CABANILLAS" en el barrio Quebracho de la ciudad de Córdoba, ubicado en el SE de Córdoba es un espacio de viviendas sociales considerado de alta vulnerabilidad social en el que participaron todos los alumnos y el plantel docente asistentes durante el período de estudio (10). La estructura del sector se caracteriza por la presencia de problemas físico- funcionales importantes, tales como falta de conectividad e integración del sector - tanto desde las relaciones internas del mismo, como desde las que mantiene con el resto de la ciudad, falta de espacios verdes e infraestructura; se suma a esto la problemática ambiental derivada de inundaciones por anegamiento y contaminación del suelo, el agua y el aire, afectando la calidad de vida integral de la población, cuya condición socio económica dominante refleja el alto grado de vulnerabilidad para enfrentar estos problemas. 
Se realizó: observación participante $(11,12)$ de los recreos y comedor, registro fotográfico de los alimentos a la venta en el kiosco (interno y externo) y de los residuos contenidos en los basureros.

Se estudiaron las siguientes variables cuantitativas: Los productos ofrecidos por el quiosco escolar, productos ofrecidos por el kiosco externo y material de desecho en los basureros de la totalidad de las aulas y de los lugares de recreo, galerías y patios. En lo que respecta a lo cualitativo se procedió a categorizar dos espacios en donde se observó consumo de alimentos, el recreo que es un espacio de distención en la jornada escolar y el comedor en donde se desarrolla la alimentación programada por el programa nutricional provincial, se tomó como participantes a los docentes y alumnos de la institución que se encontraban al momento de realizar las observaciones.

El trabajo de campo fue realizado por dos integrantes del equipo de investigación y estudiantes de odontología, conformando 2 sub equipos para abarcar ambos turnos de la jornada escolar. Previo a realizar el trabajo de campo se llevaron a cabo capacitaciones en el grupo de investigación acerca de técnicas cualitativas, al igual que la reflexión sobre los criterios de recolección de datos por parte de los profesionales integrantes.

Los datos cuantitativos se clasificaron según: gráfica de la alimentación diaria de las "Guías Alimentarias para la Población Argentina (13) y se obtuvieron medidas de frecuencias, porcentajes y se graficaron resultados, utilizando el software Excel 2010.

Con respecto a los datos cualitativos se realizó una codificación axial $(11,12)$ que permite trabajar materiales simbólicos u observacionales agrupándolos en distintas categorías que responden a una conceptualización más general, tendiente a manifestar las condiciones y los elementos referenciales del contexto. Se procedió a analizar todos los registros observacionales, dividiendo el material en dos categorías recreo y comedor y dos indicadores para estos (consumo de alimentos por parte de los alumnos y docentes en los recreos en donde se agregó la pauta de observación sobre los mismos). El atravesamiento axial que se busco es entender el consumo de alimentos entre los actores mencionados anteriormente. En consideración a ello, el registro fue abordado por los investigadores, con esas dos referencias, con lo cual se obtuvo un "quantum" de material que se debe procesar de manera sistemática y coordinada con los lineamientos del proyecto que le da origen y función, como así también con aspectos técnicos y conceptuales para potenciar los resultados obtenidos.

La manera de llevar a cabo el primer procesamiento, valoración y reducción de datos fue por medio de la elección de la codificación axial (análisis de tipo cualitativo, tendiente a manifestar las condiciones de contexto y los elementos referenciales del mismo).

Tal estrategia técnica es favorable a las instancias de diagnósticos, relevamientos y demarcaciones de situaciones, lugares, conductas generales, etc. La pertinencia de la codificación axial se debe a que los registros llevados a cabo permiten presentar al contexto en términos de futuras dimensiones de análisis teóricas e indicadores operacionales para intervenciones y proyecciones más complejas sobre el contexto o sus actores.

El objetivo demarcado fue el de identificar la preferencia de los niños en el consumo de alimentos en una semana, observado en dos instancias de registro: comedor y recreo. Estas dos últimas se convierten en las categorías axiales por las cuales se procedió a identificar indicadores de las mismas apelando a los códigos contextuales (cómo, dónde y porqué). Estos códigos ordenan el material y filtran el mismo para abordar cada categoría y sostener el principio de inclusividad/exclusividad que las categorías deben tener.

\section{Resultados}

En cuanto a la oferta del kiosco interno, se observó que de los productos que ofrece y teniendo en cuenta la gráfica de la alimentación diaria de las "Guías Alimentarias para la Población Argentina (13), y los grupos de alimentos que la conforman se registró que 
el $72,72 \%$ de los productos que estaban a la venta en el kiosco de la escuela, pertenecen al grupo donde se agrupan las azúcares y los dulces; el $18,18 \%$ al $1^{\circ}$ grupo de cereales, legumbres y derivados y el 9,09\% al de grasas y aceites. (Gráfico 1).

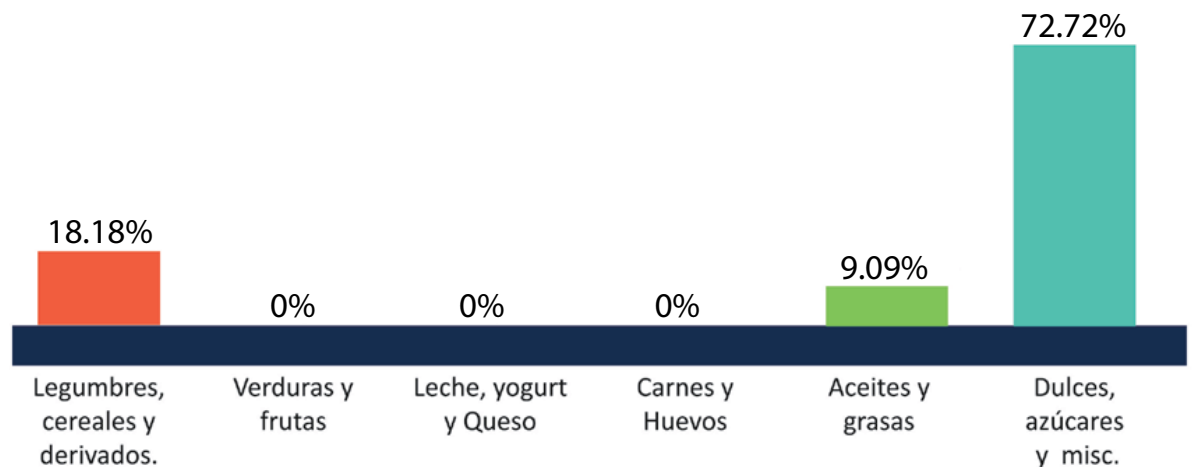

Gráfico $\mathrm{N}^{\mathrm{o}}$ 1: Distribución de la oferta de alimentos del kiosco escolar según grupos alimentarios.

Tomado de gráfica de la alimentación diaria de las "Guías Alimentarias para la Población Argentina

De los productos que se vendieron, durante los recreos en ambos turnos, se destacó una mayor venta durante el turno tarde.

Entre los productos que se vendieron durante la semana, existe una relación de 1:9 entre el grupo de alimentos de cereales, legumbres y derivados y de golosinas, dulces y azúcares) El kiosco externo, ubicado al frente de la escuela, en una casa de familia; a diario abre desde las 12 del mediodía hasta las $13,30 \mathrm{hs}$, horario en que ingresan los chicos del turno tarde, que son los que generalmente compran antes de ingresar a la escuela, según refiere el kiosquero. En cuanto a la distribución según la gráfica de la alimentación diaria de las "Guías Alimentarias para la Población Argentina el mayor porcentaje lo llevan los alimentos perteneciente al grupo de dulces y azúcares con el 62,96\%; le siguen los del grupo de cereales, legumbres y derivados con el $22,22 \%$, y el $14,81 \%$ para las grasas y aceites. (Gráfico 2).

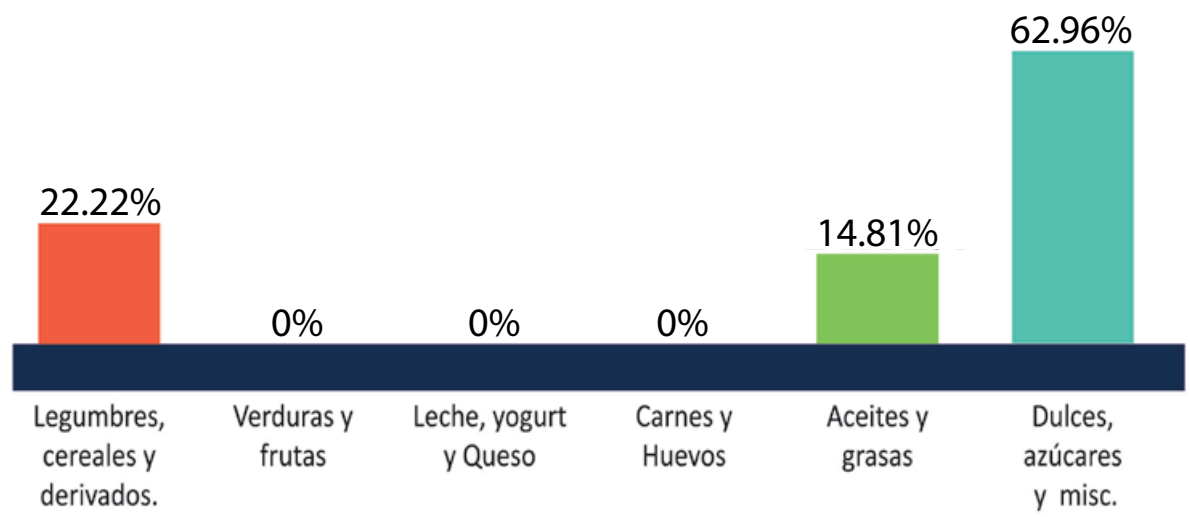

Gráfico N²: Distribución de la oferta de alimentos del kiosco externo según grupos alimentarios

Tomado de gráfica de la alimentación diaria de las "Guías Alimentarias para la Población Argentina.

Analizando el registro fotográfico de los basureros durante la semana, en ambos turnos se identificó, que de los 1.395 productos hallados, el mayor porcentaje pertenece al grupo de dulces, golosinas y azúcares con el 52,04\%, agregándose el grupo de fruta y verduras con el $26,95 \%$, los restos de fruta fueron representativos porque 4 días de la semana se 
entregaron frutas en el comedor como postre, y los niños las consumen durante los recreos. En el turno tarde, también se destaca el grupo de lácteos (leche chocolatata en envase tetra pack) con el 0,21\%. Les siguen restos de alimentos del grupo de cereales, legumbres y derivados con el 15,12\%, el grupo de grasas y aceites con el 5,66\%. (Gráfico 3).

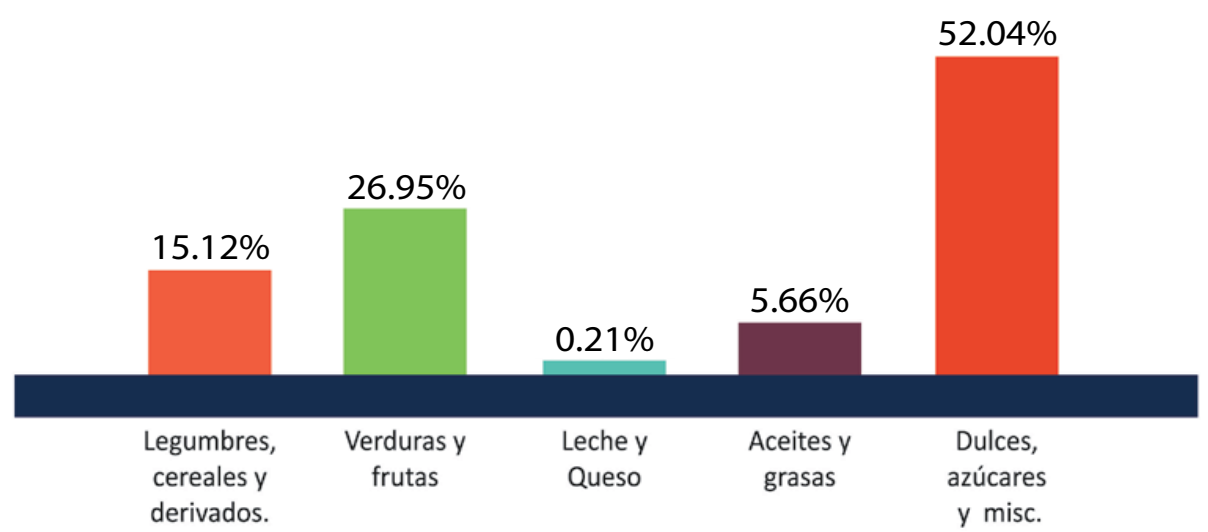

Gráfico No3: Distribución de los restos alimentarios encontrados en los basureros según grupos alimentarios

Tomado de la gráfica de la alimentación diaria de las "Guías Alimentarias para la Población Argentina

Las características de los registros cualitativos se basaron en la observación de diferentes instancias temporales que se desarrollan en la mencionada institución escolar, siendo factible definir como tales al recreo y el momento de almuerzo de los alumnos.

Durante el recreo el consumo por parte de los niños son alimentos del tipo golosinas (alfajores, chupetines, caramelos, etc.) y bebidas gaseosas y jugos en polvo preparados en la escuela, también se destacó alimentos caseros como tartas de manzanas. En esta instancia, lo docentes consumían alimentos similares a los niños, agregándose pan criollo y frutas, con respecto a la bebida (infusiones como el mate y yogurt).

En el comedor, los niños mas grandes consumían preferentemente carnes (milanesas y hamburguesas) y los pequeños (alimentos blandos como puré), y todos consumían barras de cereales y la bebida para todos era el agua.

La categoría recreo, es más densa que la de comedor, tal propiedad tal vez se deba a que en ella el registro es más libre y heterogéneo que en el comedor, siendo posible por ejemplo que el niño controle sus conductas a la vez que tiene mayor probabilidad de hacer lo que quiera o pueda, por ejemplo consumir determinado alimento o bebida de preferencia.

Se observa que los mismos en ambas categorías axiales se agrupan en torno a preferencias individuales de los niños, en particular a lo que hace a dulces, gaseosas y snacks (por ejemplo en el comedor, cuando se come un determinado alimento, generalmente frutas o flan) o, en el recreo las golosinas y gaseosas.

Por último, se debe tener en cuenta que se desprende del análisis:

-que el alimento tiene dos presentaciones ante el niño: una libre (ofertada) como en el recreo, en donde el niño de alguna manera ejerce con mayor posibilidad la preferencia; y otra reglada (en el comedor) en donde el niño selecciona en una primera instancia por la afinidad con el alimento, que en suposición contempla las necesidades nutricionales del niño pero no sus "preferencias".

-que hay alimentos que se repiten en consumo tanto para niños como para docentes en el recreo, lo cual de alguna manera estaría presentándose como un refuerzo sistemático a ese consumo del alumno, provocando una dieta de alimentos preferidos en competencia ventajosa con la ofrecida en el comedor escolar. 


\section{Discusión y Conclusión}

La alimentación en el ámbito escolar es un tópico que ha tenido formas de abordaje y análisis diferentes de acuerdo a la disciplina que lo toma. Al respecto, desde un punto de vista nutricional pueden observarse las calorías o el índice alimentario de cada producto, teniendo en cuenta la edad y el sexo del niño; desde los análisis sociológicos se ha puesto el vector de análisis en el acceso a la alimentación infantil y el rol mediatizador de la institución escolar; desde la psicología se ha hecho foco en temas como la alimentación y el desarrollo de tareas, la interiorización de pautas alimentarias o la representación que tiene el alimento para el niño o los docentes.

El consumo como comportamiento individual o colectivo es también una forma de analizar la calidad de alimento a la que acceden los niños en diferentes contextos escolares.

Los resultados mostraron un desde un punto de vista cuantitativo una tendencia de alto predominio de azúcares y golosinas con algunas diferencias importantes. En cuanto a la oferta, en el kiosco externo es más diversificado mientras que es alta la concentración de azúcares y golosinasen el kiosco interno. Esta situación es motivada por diferentes factores estructurales (recursos económicos, humanos, espacio físico, etc.) y falta de información sobre alimentación saludable y no saludable. Esto coincide en que la percepción de salud en relación a la alimentación puede guardar relación con los recursos económicos con los cuales se cuenta (14).

En el consumo, si bien hay predominio de azucares, golosinas y dulces, importante presencia tienen las frutas que provee el comedor, especialmente para el turno de la tarde, lo que demuestra que ante una oferta saludable los niños optan por ella (15).

Desde el análisis cualitativo se halló que: la categoría axial recreo es más densa en datos que comedor en preferencias alimentarias. Esto significa que los resultados están más visibles en la instancia de recreo, es decir, que los niños consumen más diversidad de alimentos que en el comedor (por elección); y que además los docentes consumen los mismos alimentos que los niños en la instancia de recreo (no observando el consumo de alimentos que hacen las maestras al momento del comedor).

Los resultados muestran una oferta de alimentos que es desequilibrada si se toman al kiosco interno (dentro de la escuela) o externo (fuera de la escuela). Tal mención no es sólo sobre los horarios en que están abiertos sino también por la oferta de productos que hacen. Considerando el turno los niños que ingresan a la tarde compran mayormente en el kiosco externo, a diferencia de los niños que ingresan a la mañana que podrían traer sus alimentos de sus casas o comprarlos en el kiosco interno que está abierto y que pueden estar mediatizados por la madre, padre o acompañante del alumno (es decir, que el niño de la mañana tiene menos autonomía para elegir el alimento que va a consumir con respecto al de la tarde).

Otra consideración es el postre que ofrece el comedor escolar, que por lo general son frutas, y que los niños consumen durante el recreo, principalmente en el turno tarde. En ocasiones el postre ofrecido eran barras de cereales y el momento de su consumo fue igual que con las frutas.

Hay alimentos que consumen tanto niños como docentes en el recreo, lo cual de alguna manera estaría presentándose como un refuerzo sistemático a ese consumo del alumno.

Teniendo en cuenta lo anterior pueden indicarse que los niños tienen ciertas relaciones con los alimentos de acuerdo a la interacción que tengan en el contexto con los adultos.

Según la figura del adulto y el tipo de relaciones afectivas que establezca con el mismo podrán reconocerse ciertos comportamientos con respecto a la alimentación (15). Si la relación o lazo emocional es positiva y profunda permitirán en el niño aprender valores y conocimientos sobre qué es lo apropiado en su alimentación según los criterios de salud de los adultos que lo rodean. Esto significa que el niño puede interiorizar como positiva una pauta de alimentación saludable, nutricionalmente óptima y socialmente 
posible, esto es, si los adultos significativos creen en preceptos positivos e importantes sobre la alimentación (por ejemplo, comer frutas por su relación con ciertos complejos vitamínicos que benefician al sistema inmunológico). Este mismo lazo positivo también puede redundar en comportamientos poco saludables al momento de la alimentación (16), como comer alimentos de alto contenido graso como papitas, chizitos, como una forma de suplementar una comida (una merienda, un desayuno).

En el mismo sentido, hay un desconocimiento del carácter negativo de algunos alimentos. En relación a ello en un estudio realizado recientemente en la Ciudad de Corrientes (Argentina) sobre el consumo de alimentos cariogénicos por parte de adultos se encontró que si bien la mayor parte de la población de estudio distingue los alimentos cariogénicos de los no cariogénicos, su consumo diario es elevado, en especial "entre las comidas principales" (17).

Esto es importante de destacar debido a que el niño en edad escolar se relaciona con adultos a los cuales se apega por constante de tiempo y de su satisfacción de necesidades (alimenticias, cognitivas, sociales, emocionales), estos contactos están representados tanto por sus padres como por los docentes que tiene la escuela; $y$ en tal sentido por medio de las relaciones de apego el niño va aprendiendo al modo de formalidades de la realidad ciertos comportamientos y la alimentación es uno de ellos, ya que el adulto formaliza el contacto y el consumo de alimentos del niño a través de la provisión del mismo y valida el consumo de acuerdo a la representación que tenga del alimento y de las necesidades del niño. Esto se ha visualizado tanto en los alimentos que las madres proponen a sus hijos ante la oferta del kiosco interno, o los consumidos por la maestra, en donde no puede diferenciarse que es lo correctamente nutricional y el alumno lo está observando directamente.

Sumado a lo anterior puede enfocarse el consumo de alimentos de los niños en etapa escolar de acuerdo a un modelo ecológico (18), este modelo prioriza una interacción dinámica del niño con su entorno el cual a modo de contexto va extendiéndose desde el más familiar hasta el más culturalmente lejano.

Con respecto a la alimentación el primer sistema determinante en el niño se denomina microsistema y está representado por la familia, en el cual el niño encuentra la satisfacción de sus necesidades básicas y la importancia que tiene la alimentación. Este sistema incluye a: la idiosincrasia familiar en lo que hace al consumo de alimentos (qué es adecuado o no) y a la accesibilidad económica de los mismos (que pueden acercar al niño a una alimentación óptima o podrían alejarlo de acuerdo a la capacidad económica de la familia. Por ejemplo, los niños traen de su casa meriendas pertenecientes a los grupos de azúcares y dulces; $y$ al de grasas y aceites

El segundo sistema en el cual puede analizarse el proceso de alimentación se denomina mesosistema representado por las relaciones sociales del grupo familiar y por el acceso a ciertas instituciones sociales, por ejemplo la escuela. La característica de este sistema es actuar como regulador entre la idiosincrasia familiar y las disposiciones del exosistema representadas por el estado y la cultura. La escuela tiene definiciones que afectan a la alimentación de un niño, ya sea que la trate como contenido pedagógico, como instancia favorecedora de adquisición de los alimentos (como comedores, kioscos, ferias de platos, etc.), y al personal de la escuela como modelo de comportamientos alimenticios. Es necesario que haya concordancia entre los contenidos pedagógicos y las prácticas alimentarias de todo el personal de la institución educativa.

También debe destacarse el exosistema, de contexto más amplio que no incluye a la persona como sujeto activo, y como posibilitador de políticas a través de programas de alimentación escolares. Los niños de sectores desfavorecidos tienen de manera prioritaria su alimentación a través de la copa de leche o el comedor escolar, accediendo de esta forma a una alimentación equilibrada). Estos programas deberían tener como punto de partida una teoría amplia sobre el consumo y comportamiento de los niños frente a los alimentos, ya que en muchas ocasiones estos programas se basan en supuestas necesidades biológicas 
de los niños marcadas por índices evolutivos y que no llegan a su objetivo porque se desconoce la regulación cognitiva y social que tiene la alimentación, por ejemplo, ofrecer en el menú escolar alimentos que el niño no come, ya sea, por preconceptos previos en su grupo familiar; o que no formen parte de los alimentos diarios que observa en su hogar; o por ser presentados de manera diferente.

En el presente trabajo se observó un marcado predominio de alimentos correspondientes al grupo 6 de la gráfica de la alimentación diaria (13), en las preferencias de consumo de los niños durante la jornada escolar. Estos resultados que se vieron enriquecido por el análisis cualitativo el cual mostró al espacio del recreo como momento más rico en la proporción de datos de las preferencias alimentarias de los escolares y el refuerzo sistemático en la elección de alimentos representado por las docentes durante la jornada escolar.

Por todo lo mencionado se propone que al momento de considerar el consumo de alimentos en niños escolarizados se tengan en cuenta las regulaciones tanto a nivel interno como externo del comportamiento de consumo de alimentos en un niño, lo cual traería como beneficio promover un comportamiento alimentario saludable desde una perspectiva más profunda y en donde se haga foco en la determinación múltiple de este comportamiento.

\section{Bibliografía}

1. Cornejo LS. Promoción contextualizada de la salud en comunidades vulnerables. Programa de Asociación de Proyectos Conjuntos de Investigación del MERCOSUR. PPCP SPU- Ministerio de Educación Nº29/2011 Períodos 2011-2013 y 2014-2015.

2. Velandia Castro N, Cardenas L, Agudelo Cely N. ¿Qué ofrecen las tiendas escolares y que consumen los estudiantes durante el descanso? Estudio en la ciudad de Tonja 2009. Revista Hacia la Promoción de la Salud. 2011; 16 (1): 87-98.

3. Theodore F, Bonvecchio Arenas A, Blanco García L, Carreto Rivera Y. Representaciones sociales relacionadas con la alimentación escolar: el caso de las escuelas públicas de la Ciudad de Mexico. Rev Salud Col. 2011; 7(2): 215-229.

4. Moncunill IA, Bella M, Cornejo L S. Consumo de alimentos durante el recreo escolar. Diseño y validación de un instrumento de relevamiento. Odontoestomatología . 2016;18(27): 30-38

5. Ministerio de Salud de la Nación [Internet]. Buenos Aires: [citado el 20 de Mar 2016]: 2016. Disponible en: www.msal.gob.ar

6. Piaggio L, Concilio C, Rolon M, Macedra G, Dupraz C. Alimentación infantil en el ámbito escolar: entre patios, aulas y comedores. Salud Colec [Internet]. 2011 [citado el 20 de Mar 2016]; 7 (2): 199-213. Disponible en: www.rsc.com .

7. Bustos N, Kain J, Leyton B. Colaciones habitualmente consumidas por niños de escuelas municipalizadas: motivaciones para su elección. Chil Nutr [Internet]. 2010 [citado el 20 Mar 2016]; 37 (2): 178-184. Disponible en: www.rcnb.com

8. Busdiecker B. Sara, Castillo D. Carlos, Salas A. Isabel. Cambios en los hábitos de alimentación durante la infancia: una visión antropológica. Rev. chil. pediatr. [Internet]. 2000 Ene [citado 2016 Nov 13]; 71(1): 5-11. Disponible en: http://www. scielo.cl/scielo.php?script=sci_arttext\&pid=S0370 41062000000100003\&lng=es. http://dx.doi.org/10.4067/S0370-41062000000100003.

9. Drewnowski A1, Mennella JA, Johnson SL, Bellisle F. Sweetness and food preference. J Nutr. 2012; 142:1142 - 1148.

10. González LM. Vulnerabilidad Educativa en el Gran Córdoba, Vulnerabilidad Social. Astrolabio. 2007; 4: 29-37.

11. Forni FH, Gallart MA, Vasilachis de Gialdino I: Métodos cualitativos II. La práctica de la investigación. Bs. As: Centro Editor de América Latina; 1992.

12. Valles M. Técnicas cualitativas de investigación social. Reflexión metodológica y práctica profesional. España: Síntesis; 1999. 
13. Ministerio de Salud de la Nación. Guías Alimentarias para la Población Argentina. Buenos Aires; 2016.

14. Anigtein MS. Alimentación de estudiantes de pregrado chilenos en el contexto de la modernidad alimentaria. Chil Nutr. 2013; 40 (3): 243-249.

15. Bowlby J, Ainswoorth M. Attachment Theory. Developmental Psychology. 1990; 28, 759-775.

16. Early Child Care Research Network. ¿Son efectivas las charlas para prevenir el aumento de peso en escolares? Revista Internacional de Medicina y la Actividad Física [Internet]. 2006 [citado 14 Feb 2016]. Disponible en: www. rediris.com.

17. Dho MS. Consumo de alimentos cariogénicos en adultos de la ciudad de Corrientes, Argentina. Hacia Promoc. Salud. 2015; 20 (2): 90-101.

18. Bronmfrenbrener, U. Ecology of the family as a context for human development: Research perspective. Development Psychology. 1986; 22 (6): 723-742. 